\section{BRAZIULIAN JOURNAL \\ OF MEDICAL AND BIOLOGICAL RESHARCH}

www.bjournal.com.br
ISSN 0100-879X

Volume 42 (8) 692-775 August 2009

CLINICAL INVESTIGATION

Braz J Med Biol Res, August 2009, Volume 42(8) 750-755

Raising awareness of chronic kidney disease in a Brazilian urban population

M. Mazza Nascimento and M.C. Riella

The Brazilian Journal of Medical and Biological Research is partially financed by
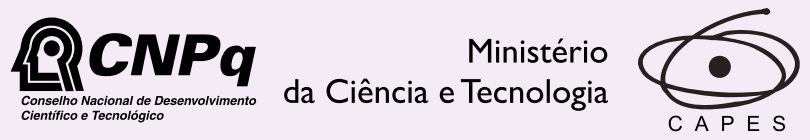

Ministério da Educação

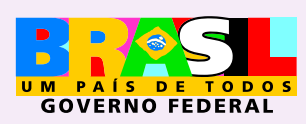

Institutional Sponsors 


\title{
Raising awareness of chronic kidney disease in a Brazilian urban population
}

\author{
M. Mazza Nascimento ${ }^{1}$ and M.C. Riella ${ }^{1,2}$ \\ 1Departamento de Nefrologia, Faculdade Evangélica de Medicina do Paraná, Curitiba, PR, Brasil \\ ${ }^{2}$ Centro de Ciências Médicas e Biológicas Pontifícia Universidade Católica do Paraná, Curitiba, PR, Brasil \\ Correspondence to: M. Mazza Nascimento, Faculdade Evangélica de Medicina do Paraná, Saldanha \\ Marinho, 1453, 80430-160 Curitiba, PR, Brasil \\ Fax: +55-41-3322-0490. E-mail: mazza@bsi.com.br
}

\begin{abstract}
The incidence and prevalence of chronic kidney disease have been increasing in recent years in developing countries. The aim of this study was to report the results of a general chronic kidney disease awareness program applied to an urban population in a large Brazilian city. From January 2002 to January 2005 a total of 8883 individuals in the city of Curitiba (PR, Brazil) were screened for hypertension, body mass index, hematuria, and proteinuria. A family history and previous medical diagnosis of hypertension and diabetes mellitus (DM) were also recorded. Of the 8883 individuals assessed, 56\% were women, subject median age was 47 years (range: $17-93$ years) and more than $90 \%$ were Caucasian. Thirty percent had signs of hematuria, $6 \%$ had proteinuria, and $3 \%$ had hematuria and proteinuria. The median of mean arterial pressure values was $93 \mathrm{mmHg}(\mathrm{range}: 71-$ $135 \mathrm{mmHg}$ ) and $16 \%$ of the population screened had a history of hypertension. A significant positive family history of both hypertension or DM was present in $42 \%(P<0.0001$; chi-square $=83.18)$ and $7 \%(P<0.0001$; chi-square $=161.31)$ of the hypertensive group, respectively. Finally, the prevalence of hypertension and DM was significantly higher in older individuals with proteinuria. In the present study, a higher prevalence of hematuria and proteinuria was found in older individuals with hypertension and diabetes compared to the general population. These data confirm the need for public awareness of renal disease in high-risk individuals.
\end{abstract}

Key words: Hematuria; Proteinuria; Prevention; Chronic kidney disease; Screening

Received September 22, 2008. Accepted May 8, 2009

\section{Introduction}

The incidence and prevalence of chronic kidney disease (CKD) have been increasing in recent years in developing countries (1). The prevalence of CKD (10-13\%) in Europe according to the PREVEND (2), EPIRCE (3) and HUNT (4) studies is similar to that reported by the Third National Health Examination Survey (NHANES III) (5) However, in developing countries, few studies have investigated the real prevalence of CKD in the general population. Data from the Latin American Society of Nephrology and Hypertension estimate that the prevalence of patients on renal replacement therapy increased from 129 per million population (pmp) in 1992 to 447 pmp in 2004 (6). More recently, the Villarica Consensus Statement highlighted the need for screening and intervention studies of high-risk population for kidney disease to establish a real estimate of CKD in Latin America (7).

In Brazil, the real prevalence of CKD is unknown. In 2003, Passos et al. (8) reported increased creatinine levels in $0.48 \%$ of a population screened from a small town in southwestern Brazil. They also reported a significant elevated creatinine plasma levels in older hypertensive individuals. Furthermore, Oliveira et al. (9) recently reported that the prevalence of end-stage renal disease in Brazil is approximately $390 \mathrm{pmp}$, which corresponds to one third of the prevalence in the USA. This finding suggests that CKD is an under-diagnosed condition in Brazil. This scenario indicates the need for programs of early identification and awareness of CKD in this area of the world. The aim of the present study was to report the results of a general chronic kidney disease awareness program applied by the ProRenal Foundation to an urban population in a large Brazilian city. 


\section{Subjects and Methods}

\section{Study design}

From January 2002 to January 2005 a total of 8883 individuals participated in the study in the city of Curitiba, the capital of the State of Paraná, which is located in southern Brazil, with an estimated population of 2 million inhabitants. Individuals younger than 13 years were excluded from the screening. The program was conducted by doctors, nurses and social workers and the main goal was to raise awareness of CKD by the detection of asymptomatic urinary abnormalities (dipstick test) and its association with the presence of hypertension, diabetes mellitus (DM) and age. Menstruating women were excluded up to three days after their period. Participants were self-referred to an existing Health Fair taking place in their area and organized by the Pro-Renal Foundation, a non-profit organization dedicated to patient assistance, education and research in kidney diseases (www.pro-renal.org.br).

All participants were weighed and had their height measured. BMI was calculated by dividing body weight by height squared $\left(\mathrm{kg} / \mathrm{m}^{2}\right)$. Blood pressure was measured with a mercury sphygmomanometer using standard methods. Measurements were made by trained and certified staff with the subject in the sitting position. Systolic and diastolic blood pressure readings were derived from the average of two measurements. Mean arterial pressure was calculated as the sum of one third the systolic reading plus two thirds the diastolic reading. Hypertension was defined as self-reported (medical diagnosis) or by the use of antihypertensive medications and DM was defined as a previous history of diabetes (self-reported) and/or by the use of medications to treat diabetes.

\section{Dipstick test}

Participants were instructed to void a clean urine specimen into an appropriate container. The analysis was based on the dipstick reagent strip, which reacts with the urine producing color modifications over time (Combur Test UX, Roche Laboratory, Germany). The results were analyzed by comparing the test strip to a color chart attached to the tube label after a careful visualization of the strip. Patients were divided into the following groups according to the result of the dipstick test: hematuria alone, proteinuria alone, and concomitant presence of hematuria and proteinuria. The proteinuria results were recorded as (-) (normal, absence of proteinuria) or $(+)$ (abnormal, presence of proteinuria) and abnormal results were graded as $1+, 2+, 3+, 4+$.

Each individual received the printed result of urinalysis and comments about abnormalities, high blood pressure, or excessive weight. Individuals were instructed to seek medical attention when indicated. Educational leaflets about DM, hypertension, and prevention of CKD were also distributed. The Ethics Committee of Hospital Evangélico de Curitiba approved the study protocol.

\section{Statistical analysis}

Data are reported as means $\pm S D$, or median and range as appropriate. Comparison between two groups was performed by the Student $t$-test for normally distributed variables, whereas the Mann-Whitney U-test was used for non-normally distributed variables. Categorical variables were analyzed using contingency tables. For non-normally distributed variables, correlations were performed with the Spearman rank tests. The difference was considered significant when $P$ was less than 0.05 .

\section{Results}

\section{Clinical data}

The clinical characteristics of the group are given in Table 1. Of the 8883 individuals assessed, $44 \%$ were men, median age of 47 (17-93 years) and more than $90 \%$ of the subjects in the study population were Caucasian. Thirty percent had hematuria, $6 \%$ had proteinuria, and $3 \%$ had hematuria and proteinuria. The median of mean arterial pressure (MAP) values was $93 \mathrm{mmHg}$ (range: $71-135 \mathrm{mmHg}$ ), $16 \%$ of the screened individuals reported a medical diagnosis of systemic hypertension, and $25 \%$ of the subjects, without a previous diagnosis of hypertension, had increased pressure rates (systolic blood pressure $\geq 140 \mathrm{mmHg}$ and diastolic blood pressure $\geq 90 \mathrm{mmHg}$ ). Moreover, more than half of the diabetic individuals were hypertensive $(P<0.001$; chi-square $=44.83)$. In the hypertensive population, $42 \%$ had a significant positive family history of hypertension $(\mathrm{P}<$ 0.0001 ; chi-square $=83.18$ ) and $7 \%$ of them were diabetics $(P<0.0001$; chi-square $=161.31)$. BMI median was $24.8 \mathrm{~kg} /$ $\mathrm{m}^{2}$ and a positive and significant correlation was found between $\mathrm{BMI}$ and MAP (Rho = 0.30; $\mathrm{P}<0.001$; Figure 1).

No significant differences were found in age, BMI, MAP, or history of DM in individuals with or without hematuria. On the other hand, hypertension, the concomitant presence of proteinuria and female gender were significantly higher in individuals with hematuria (Table 2). Proteinuria was significantly associated with age and hematuria (Table 3). In addition, proteinuria was significantly higher in hypertensive $(P<0.001$; chi-square $=43.14)$ and diabetic patients $(P<0.001$; chi-square $=44.83$ ) and more than half of the diabetic individuals were hypertensive $(\mathrm{P}<$ 0.001; chi-square $=44.83$ ). However, neither BMI nor gender was associated with the presence of proteinuria. Finally, older people (age >60 years) had significantly 


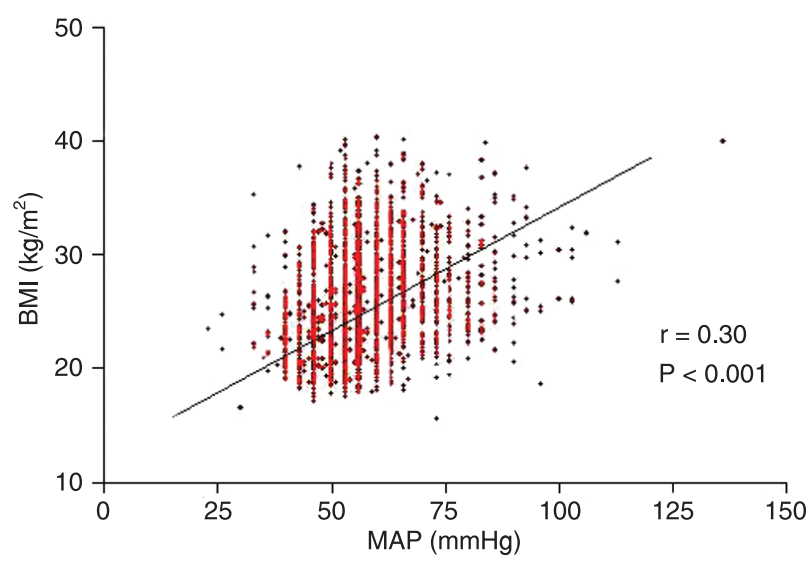

Figure 1. Correlation between body mass index (BMI) and mean arterial pressure (MAP).

Table 1. Clinical characteristics and urine abnormalities of the screened population.

\begin{tabular}{lc}
\hline & $\begin{array}{c}\text { Screened population } \\
(\mathrm{N}=8883)\end{array}$ \\
\hline Age (years) & $48 \pm 16$ \\
Male gender & $44 \%$ \\
Hypertension & $16 \%$ \\
Diabetes mellitus & $3 \%$ \\
Hematuria & 30 \\
Body mass index $\left(\mathrm{kg} / \mathrm{m}^{2}\right)$ & $24.8(17-40)$ \\
Mean arterial pressure $(\mathrm{mmHg})$ & $93(71-135)$ \\
Proteinuria & $6 \%$ \\
Glycosuria & $4 \%$ \\
Hematuria and proteinuria & $3 \%$ \\
\hline
\end{tabular}

Data are reported as means $\pm S D$, percent or median (range).

Table 2. Clinical characteristics and dipstick abnormalities in individuals with hematuria.

\begin{tabular}{lcc}
\hline & $\begin{array}{c}\text { Hematuria } \\
(\mathrm{N}=2665)\end{array}$ & $\begin{array}{c}\text { Non-hematuria } \\
(\mathrm{N}=6218)\end{array}$ \\
\hline Age (years) & $49(17-89)$ & $45(17-93)$ \\
$\mathrm{BMI}\left(\mathrm{kg} / \mathrm{m}^{2}\right)$ & $25(18-40)$ & $24(17-40)$ \\
Females & $72 \%^{*}$ & $49 \%$ \\
MAP $(\mathrm{mmHg})$ & $90(71-126)$ & $93(71-135)$ \\
Proteinuria & $10 \%^{*}$ & $3 \%$ \\
Hypertension & $12 \%^{*}$ & $9 \%$ \\
Diabetes mellitus & $3 \%$ & $2 \%$
\end{tabular}

Data are reported as median (range) or percent. $\mathrm{BMI}=$ body mass index; MAP = mean arterial pressure. ${ }^{*} \mathrm{P}<0.05$ compared to nonhematuria group (chi-square test and Mann-Whitney U-test). higher BMI, proteinuria, hematuria, hypertension, and DM compared to the younger population (Table 4).

\section{Discussion}

The main finding of the present study was a significantly higher prevalence of proteinuria and hematuria in older individuals with hypertension and/or DM. The prevalence of proteinuria in the current study was $6 \%$. This finding agrees with other studies, performed in Bolivia and Japan, in which the prevalence of proteinuria ranged from 4 to $6 \%$ $(10,11)$. The importance of detecting proteinuria in the general population is relevant. In fact, proteinuria is a reliable marker of the severity of CKD and a powerful and independent predictor of its progression $(12,13)$. We also observed that the presence of proteinuria was more evident in the high-risk population (older, diabetic, and hypertensive patients). Several literature reports (14-16) have

Table 3. Clinical characteristics and dipstick abnormalities of individuals with proteinuria.

\begin{tabular}{lcc}
\hline & $\begin{array}{c}\text { Proteinuria } \\
(\mathrm{N}=533)\end{array}$ & $\begin{array}{c}\text { Non-proteinuria } \\
(\mathrm{N}=8350)\end{array}$ \\
\hline Age (years) & $54(17-91)^{*}$ & $45(17-93)$ \\
$\mathrm{BMI}\left(\mathrm{kg} / \mathrm{m}^{2}\right)$ & $25(17-36)$ & $24(17-40)$ \\
Females & $53 \%$ & $56 \%$ \\
MAP $(\mathrm{mmHg})$ & $93.3(71-126)^{*}$ & $92.6(71-135)$ \\
Hematuria & $56 \%^{*}$ & $27 \%$ \\
Hypertension & $12 \%^{*}$ & $9 \%$ \\
Diabetes mellitus & $5 \%^{*}$ & $2 \%$ \\
\hline
\end{tabular}

Data are reported as median (range) or percent. $\mathrm{BMI}=$ body mass index; MAP = mean arterial pressure. ${ }^{*} \mathrm{P}<0.05$ compared to non-proteinuria group (chi-square test and Mann-Whitney $U$ test).

Table 4. Clinical characteristics and dipstick abnormalities according to age.

\begin{tabular}{lcc}
\hline & $>60$ years & $<60$ years \\
& $(\mathrm{N}=2608)$ & $(\mathrm{N}=6275)$ \\
\hline BMI $\left(\mathrm{kg} / \mathrm{m}^{2}\right)$ & $27(16-40)^{*}$ & $25(17-40)$ \\
Females & $53 \%$ & $55 \%$ \\
Proteinuria & $9 \%^{*}$ & $5 \%$ \\
Hematuria & $31 \%^{*}$ & $29 \%$ \\
Hypertension & $62 \%^{*}$ & $33 \%$ \\
Diabetes mellitus & $7 \%^{*}$ & $2 \%$
\end{tabular}

Data are reported as median (range) or percent. $\mathrm{BMI}=$ body mass index. ${ }^{*} \mathrm{P}<0.05$ compared to $<60$ years group (chi-square test and Mann-Whitney U-test). 
pointed out in particular the association between proteinuria, microalbuminuria and cardiovascular disease in diabetic patients. However, this association is less clear in non-risk populations. In the Framingham Heart Study (17), proteinuria, detected by dipstick, was an independent marker of cardiovascular mortality in women but not in men. More recently, Brantsma et al. (18) reported that urinary albumin excretion is a powerful predictor of cardiovascular events during the first 5 years of follow-up in a community-based population.

Regarding CKD progression, Iseki et al. (11) observed that, compared to hematuria, proteinuria was a more potent predictor of CKD progression, with a cumulative $7.1 \%$ incidence of CKD in screened persons with a 2+ proteinuria score. They also reported that proteinuria was a strong and independent predictor of mortality in a mass screening setting. Furthermore, Boulware et al. (19) demonstrated that the early detection of proteinuria, aimed at slowing the progression of CKD and decreasing mortality, was not cost-effective unless selectively directed toward high-risk groups (hypertensive and diabetic populations). In the current study, the presence of hematuria (30\%) in the screened population as a whole was very high. Hematuria is more common in women, as also reported by others (20). Plata et al. (10) studied the prevalence of renal disease in subjects with urinary abnormalities by the analysis of fresh urine in a program of renal disease screening in Bolivia. These investigators showed that hematuria and urinary tract infection were by far the most common urinary abnormalities detected. However, a confirmatory second analysis did not confirm the presence of hematuria in $35 \%$ of the subjects. Similarly, the high prevalence of hematuria detected in the present study should be confirmed in a second urine analysis. The combination of hematuria and proteinuria is a powerful predictor of significant parenchymal disease (21). Yamagata et al. (22) demonstrated that $14.9 \%$ of a screened asymptomatic population with this urinary abnormality developed CKD within 5.8 years of follow-up. Although the cited study showed that patients with pure hematuria did not exhibit renal insufficiency, $10.6 \%$ of them were proteinuric during the follow-up period. Therefore, careful observation and follow-up are needed for these patients.

It has been reported that the prevalence of hypertension in Latin America reaches almost 30\%, although more than half of the patients are unaware of this condition (23). $\mathrm{Hsu}$ et al. (24) showed that even a relatively modest elevation in blood pressure was an independent risk factor for CKD and the early recognition of this condition should be part of strategies for CKD prevention. It is interesting to note that in the present investigation $25 \%$ of the studied population, without a previous diagnosis of hypertension, had increased blood pressure rates. It is important to stress that this diagnosis needs further confirmation since blood pressure was measured on only a single occasion and in the presence of the "white coat" effect. These individuals need to be aware of the risks of hypertension and at the same time should be referred for medical followup to confirm or not the presence of hypertension.

Non-insulin-dependent DM is the major contributor to the increase of CKD throughout the world. On average, $30 \%$ of diabetics will develop nephropathy (25). There is an estimate of 19 million patients with this condition in Latin America (23). The participants in the present study who reported having DM also had a higher prevalence of hypertension, proteinuria and a higher BMI (data not shown), and were significantly older. These results illustrate the need to provide to this special group of patients more information about their risk of kidney and cardiovascular disease, which requires a public health prevention strategy.

It has been a source of debate which screening strategy should be used to detect CKD patients: specific target individuals (subjects with hypertension, DM and cardiovascular disease) rather than the general population should be screened for CKD detection in screening programs (26). It is important to stress that in the present study the screened population was self-referred to our fairs. Therefore, these individuals were interested in becoming aware of their kidney disease, a fact that may not reflect the real prevalence of kidney disease in the general population. It has been reported that, for every patient with known hypertension and DM, there is an individual for whom the diagnosis has not been made (27).

Our study had several limitations. First is the fact that the screened subjects were individuals who showed concern about their general health, and they should therefore be considered as a self-selected population. Second, proteinuria, hematuria and other laboratory variables were determined only once. Orthostatic proteinuria, diet, infection, menstruation, transient fever, hydration status, and physical activity may affect the level of protein and blood in urine. In the NHANES III survey, only $63 \%$ of the initially positive results of microalbuminuria could be confirmed on repeat testing (28). Third, neither microalbuminuria nor creatinine was measured to determine more precisely the extension of kidney disease. Finally, most screened individuals were Caucasian, a fact that may not be representative of Brazilian ethnicity compared to other Brazilian areas where the prevalence of Caucasians is lower (29).

The present study describes the strategy adopted to raise awareness about CKD in a community and the pre- 
liminary results obtained. Although we understand the limitations for the interpretation of our results, mainly related to the self-referred process, we believe this is a valid strategy. The National Kidney Foundation Early Evaluation Program (KEEP) (30) targets a population with the highest risk of developing CKD, i.e., those with a high risk of hypertension and diabetes. On the other hand, the NHANES III (5) series is a cross-sectional survey monitoring the health and nutritional status of the non-institutionalized civilian population in the United States. While the KEEP survey targets a high-risk population and the NHANES III targets randomly selected individuals in the community, our strategy uses the "convenient sample"

\section{References}

1. Meguid El Nahas A, Bello AK. Chronic kidney disease: the global challenge. Lancet 2005; 365: 331-340.

2. Atthobari J, Gansevoort RT, Visser ST, de Jong PE, de Jong-van den Berg LT. The effect of screening for cardiorenal risk factors on drug use in the general population. $\mathrm{Br} \mathrm{J}$ Clin Pharmacol 2007; 64: 810-818.

3. Otero A, Gayoso P, Garcia F, de Francisco AL. Epidemiology of chronic renal disease in the Galician population: results of the pilot Spanish EPIRCE study. Kidney Int Suppl 2005; S16-S19.

4. Hallan SI, Coresh J, Astor BC, Asberg A, Powe NR, Romundstad $S$, et al. International comparison of the relationship of chronic kidney disease prevalence and ESRD risk. J Am Soc Nephrol 2006; 17: 2275-2284.

5. Coresh J, Astor BC, Greene T, Eknoyan G, Levey AS. Prevalence of chronic kidney disease and decreased kidney function in the adult US population: Third National Health and Nutrition Examination Survey. Am J Kidney Dis 2003; 41: 1-12.

6. Cusumano AM, Di Gioia C, Hermida O, Lavorato C. The Latin American Dialysis and Renal Transplantation Registry Annual Report 2002. Kidney Int Suppl 2005; S46-S52.

7. Dirks JH, Robinson S, Burdmann E, Correa-Rotter R, Mezzano S, Rodriguez-Iturbe B. Prevention strategies for chronic kidney disease in Latin America: a strategy for the next decade - a report on the Villarica Conference. Ren Fail 2006; 28: 611-615.

8. Passos VM, Barreto SM, Lima-Costa MF. Detection of renal dysfunction based on serum creatinine levels in a Brazilian community: the Bambui Health and Ageing Study. Braz J Med Biol Res 2003; 36: 393-401.

9. Oliveira MB, Romao JE Jr, Zatz R. End-stage renal disease in Brazil: epidemiology, prevention, and treatment. Kidney Int Suppl 2005; S82-S86.

10. Plata R, Silva C, Yahuita J, Perez L, Schieppati A, Remuzzi $G$. The first clinical and epidemiological programme on renal disease in Bolivia: a model for prevention and early diagnosis of renal diseases in the developing countries. Nephrol Dial Transplant 1998; 13: 3034-3036.

11. Iseki K, Ikemiya $Y$, Iseki $C$, Takishita S. Proteinuria and the approach through the promotion of health fairs in the community. We believe that programs such as ours also help raise awareness in the community for risk factors of chronic kidney disease. Non-governmental organizations such as our Kidney Foundation can amplify the health care activities of local governments, particularly in the prevention arena.

\section{Acknowledgments}

We thank the Pro-Renal Foundation for devising, implementing, and supporting this prevention program on a continuous basis. risk of developing end-stage renal disease. Kidney Int 2003; 63: 1468-1474.

12. Schieppati A, Perico N, Remuzzi G. Preventing end-stage renal disease: the potential impact of screening and intervention in developing countries. Kidney Int 2003; 63: 19481950.

13. Schoolwerth AC, Engelgau MM, Hostetter TH. A public health action plan is needed for chronic kidney disease. Adv Chronic Kidney Dis 2005; 12: 418-423.

14. Gerstein HC, Mann JF, Yi Q, Zinman B, Dinneen SF, Hoogwerf B, et al. Albuminuria and risk of cardiovascular events, death, and heart failure in diabetic and nondiabetic individuals. JAMA 2001; 286: 421-426.

15. Agewall S, Wikstrand J, Ljungman S, Fagerberg B. Usefulness of microalbuminuria in predicting cardiovascular mortality in treated hypertensive men with and without diabetes mellitus. Risk Factor Intervention Study Group. Am J Cardiol 1997; 80: 164-169.

16. Miettinen H, Haffner SM, Lehto S, Ronnemaa T, Pyorala K, Laakso M. Proteinuria predicts stroke and other atherosclerotic vascular disease events in nondiabetic and non-insulin-dependent diabetic subjects. Stroke 1996; 27: 20332039.

17. Culleton BF, Larson MG, Parfrey PS, Kannel WB, Levy D. Proteinuria as a risk factor for cardiovascular disease and mortality in older people: a prospective study. Am J Med 2000; 109: 1-8.

18. Brantsma AH, Bakker SJ, de Zeeuw D, de Jong PE, Gansevoort RT. Extended prognostic value of urinary albumin excretion for cardiovascular events. J Am Soc Nephrol 2008; 19: 1785-1791.

19. Boulware LE, Jaar BG, Tarver-Carr ME, Brancati FL, Powe NR. Screening for proteinuria in US adults: a cost-effectiveness analysis. JAMA 2003; 290: 3101-3114.

20. Santa Cruz F, Cabrera W, Barreto S, Mayor MM, Baez D. Kidney disease in Paraguay. Kidney Int Suppl 2005; S120S125.

21. Topham PS, Jethwa A, Watkins M, Rees Y, Feehally J. The value of urine screening in a young adult population. Fam Pract 2004; 21: 18-21. 
22. Yamagata K, Yamagata $Y$, Kobayashi M, Koyama A. A long-term follow-up study of asymptomatic hematuria and/ or proteinuria in adults. Clin Nephrol 1996; 45: 281-288.

23. Rodriguez-Iturbe B, Bellorin-Font E. End-stage renal disease prevention strategies in Latin America. Kidney Int Suppl 2005; S30-S36.

24. Hsu CY, McCulloch CE, Iribarren C, Darbinian J, Go AS. Body mass index and risk for end-stage renal disease. Ann Intern Med 2006; 144: 21-28.

25. Sedor JR. Frontiers in diabetic nephropathy: can we predict who will get sick? J Am Soc Nephrol 2006; 17: 336-338.

26. Levey AS, Atkins R, Coresh J, Cohen EP, Collins AJ, Eckardt $\mathrm{KU}$, et al. Chronic kidney disease as a global public health problem: approaches and initiatives - a position statement from Kidney Disease: Improving Global Outcomes.
Kidney Int 2007; 72: 247-259.

27. de Jong PE, van der Velde M, Gansevoort RT, Zoccali C. Screening for chronic kidney disease: where does Europe go? Clin J Am Soc Nephrol 2008; 3: 616-623.

28. Atkins RC. The epidemiology of chronic kidney disease. Kidney Int Suppl 2005; S14-S18.

29. http://www.ibge.gov.br/home/presidencia/noticias/ noticia_impressao.php?id_noticia $=737$. Accessed January 15,2008

30. Whaley-Connell AT, Sowers JR, Stevens LA, McFarlane SI, Shlipak MG, Norris KC, et al. CKD in the United States: Kidney Early Evaluation Program (KEEP) and National Health and Nutrition Examination Survey (NHANES) 19992004. Am J Kidney Dis 2008; 51: S13-S20. 